\title{
Características Socioeconómicas y Rendimiento Académico. El Caso de una Universidad Argentina
}

\author{
Socioeconomic Background and Academic Achievement. \\ The Case of an Argentine University
}

\author{
Carlos Cristóbal Coschiza * \\ Juan Martín Fernández \\ Guillermo Gapel Redcozub \\ Marcelo E. Nievas \\ Haraví E. Ruiz \\ Universidad Nacional del Nordeste
}

\begin{abstract}
El objetivo de la investigación es determinar mediante regresiones estadísticas las relaciones entre las características socioeconómicas de los alumnos de las carreras de grado de la Universidad Nacional del Nordeste (UNNE, Argentina) y sus resultados académicos, y compararlos con los hallazgos de estudios similares efectuados en otras universidades nacionales. Se encuentra evidencia contundente de que las variables de Nivel Socioeconómico (NSE) son significativas para explicar las diferencias de rendimiento académico entre los alumnos de las facultades analizadas. Las variables que se han utilizado como indicadores de NSE elevado han presentado una correlación positiva, y las variables que se emplearon como indicadores de NSE más bajo han presentado una correlación negativa. Las variables sexo femenino, edad, estado civil casado y situación laboral inactiva están asociadas a un mejor rendimiento académico, mientras que las variables cantidad de hijos del estudiante, situación laboral ocupado, situación laboral desocupado, ausencia de cobertura médica y beneficiario de planes sociales están relacionadas a menores desempeños académicos. En materia de género, el hallazgo más relevante se manifiesta en los resultados de las estudiantes mujeres con hijos: su rendimiento es notoriamente inferior al de los estudiantes hombres con hijos.
\end{abstract}

Descriptores: Políticas educativas, Educación superior, Características socioeconómicas, Rendimiento escolar, Estudio de casos.

The aim of our research is to assess the relationships between socioeconomic characteristics of students in undergraduate courses of Northeastern University (UNNE, Argentina) and their academic results using statistical regression, and to compare the findings with the ones obtained in similar studies in other national universities. There is strong evidence that variables of Socioeconomic Status (SES) are significant in explaining the differences in academic achievement between students of the several schools. The variables used as indicators of high SES presented a positive correlation, and variables that were used as indicators of lower SES have presented a negative correlation. The variables female sex, age, married marital status and inactive employment status are associated with better academic performance, while the variables number of children of the student, busy employment status, employment status, medical coverage, and beneficiary of social plans are related to lower academic performance. Regarding gender, the most important finding is that female students with children have lower performance than male students with children.

Keywords: Educational policy, Higher education, Academic achievement, Socioeconomic background, Latin America, Case studies.

*Contacto: carloscoschiza@gmail.com

ISSN: $1696-4713$

www.rinace.net/reice/

revistas.uam.es/reice
Recibido: $\quad 15$ de abril 2015

$1^{\text {a }}$ Evaluación: 25 de julio 2015

$2^{\text {a }}$ Evaluación: 3 de enero 2016

Aceptado: 17 de febrero 2016 


\section{Introducción}

Las investigaciones sobre rendimiento académico reconocen como antecedente fundamental al estudio sobre "Igualdad de Oportunidades Educativas" realizado en Estados Unidos a mediados de 1960 por James Coleman (1966, 1967a, 1967b, 1968a, 1968b). Desde entonces, los investigadores en el área de la educación han dedicado intensos esfuerzos para ratificar, corregir o rebatir sus hallazgos acerca de cuáles son los factores determinantes del desempeño escolar. Estas discusiones generaron una copiosa bibliografía que incluye estudios realizados desde diferentes ópticas, con diversas metodologías, en distintas áreas de conocimiento y para todas las etapas educacionales, aunque poniendo mayor énfasis en las primeras instancias de los sistemas educativos (escuelas primarias).

El presente trabajo recoge estos antecedentes con la intención de mejorar la comprensión de las variables con incidencia en el rendimiento académico de un determinado grupo de alumnos. El objetivo central de la investigación es determinar las relaciones entre las características socioeconómicas de los alumnos de las carreras de grado de la Universidad Nacional del Nordeste (UnNE, Argentina) y sus resultados académicos, y compararlos con los hallazgos de estudios similares efectuados en otras universidades nacionales.

En estos términos, resultaría válido considerar a la investigación como un "análisis de caso”, destacando que la Universidad Nacional del Nordeste es una institución pública, no arancelada, de escala media dentro del sistema universitario argentino (tiene aproximadamente 50.000 alumnos matriculados), y que ofrece numerosas carreras de grado por medio de sedes situadas en diversas ciudades del nordeste argentino.

La información provista por esta investigación podría resultar de utilidad para la creación de políticas educativas universitarias que contribuyan tanto a al incremento de la eficacia académica como a la igualdad de oportunidades educativas, y que estén específicamente diseñadas teniendo en cuenta las características y las necesidades particulares de los alumnos de la institución analizada.

\section{Revisión de la literatura}

El Reporte Coleman (Coleman et al., 1966) fue el estudio que marcó un hito en la investigación macro social en eficacia educativa [véase Cervini (2002a) o Scheerens (2000) para una revisión extensiva de los antecedentes]. Este trabajo trascendió la consigna inicial de relevar los niveles de segregación racial en las escuelas, y se abocó al estudio de los factores familiares y escolares que explican las diferencias de rendimiento de los alumnos. Sus resultados fueron muy controvertidos: la escuela con sus recursos no parecía tener mayor influencia en el aprendizaje de los alumnos, una vez que se tenía en cuenta sus características socioeconómicas. Esto sembró un profundo cuestionamiento sobre el rol de la escuela como institución democratizadora y creadora de oportunidades educativas homogéneas, convirtiéndola en cambio en un engranaje del mecanismo de reproducción cultural de las clases sociales impulsado desde el mismo estado. Jencks (1972) respalda estos resultados.

Las reacciones en contra del Reporte no se hicieron esperar. En el orden metodológico se cuestionó la simplicidad del modelo utilizado (de insumo-producto) y se objetó la forma empleada para diseñar y medir algunas variables, en especial las escolares o académicas 
(véase Sorensen, Dumais y Stephen, 1999). En el orden teórico, se criticó la ausencia de un modelo conceptual y relacional concreto con base en el cual desarrollar cualquier empresa empírica y poder descartar el efecto de variables omitidas en determinados casos.

No obstante, a partir del Reporte se generaron una gran cantidad de investigaciones que analizaban el fenómeno con distintas metodologías empíricas, al punto que hacia fines de los años ochenta se hicieron frecuentes las investigaciones con la técnica de meta-análisis, técnica que consiste en analizar un gran número de investigaciones y sus resultados, sintetizándolas. Las más importantes (Fraser, Walberg, Welch y Hattie, 1987; Hanushek, 2003; Walberg, 1986) coincidían en afirmar que "los antecedentes familiares del alumno se asocian significativamente con el rendimiento, y si bien su peso relativo puede igualar al de otros factores, difícilmente será inferior" (Cervini, 2002a, p. 450).

Las investigaciones se expandieron a los países en desarrollo, en estudios como los de Heyneman (1976), Heyneman y Loxley (1982) y Fuller (1987). A partir de ellos empezó a tomar fuerza la idea de que en los países en desarrollo los efectos de las variables escolares en el rendimiento educativo de los estudiantes primarios son significativos. Esta posición también recibió críticas, que hacen hincapié en dificultades metodológicas y de medición (Vélez, Schiefelbein y Valenzuela, 1994), agregándose además que los efectos diferenciales en los países en desarrollo pueden haber desaparecido tras las mejoras que se produjeron en sus sistemas educativos (Baker, 2002, citado por Blanco, 2009).

Al estudiar la cuestión en Latinoamérica (Argentina, Chile, Uruguay y México), Fernández Aguerre (2004) halló evidencia de que "no existía una única estructura de determinantes común entre los países” (p. 537). Es decir que en los distintos países latinoamericanos, el efecto y la importancia del conjunto de variables asociadas al rendimiento escolar no se ha mantenido constante. En el mismo sentido, Murillo (2003) sostiene que:

\section{(...) los factores de eficacia escolar encontrados en las distintas investigaciones han mostrado que, junto con los hallados en investigaciones de otros países, aparecen otros elementos propios de nuestro contexto (...). De esta forma, se puede concluir que los resultados de la investigación internacional sobre eficacia escolar resultan una excelente base, un punto de partida, para la realización de trabajos en Iberoamérica. Sin embargo, han de ser complementados con informaciones procedentes de investigaciones y análisis generados de la propia realidad iberoamericana. (p. 10, el destacado es propio)}

Estas afirmaciones implicarían que cada país, y aún cada región dentro del mismo país, debería identificar cuál es su propia matriz de determinantes del rendimiento educativo y elaborar políticas educacionales acordes a sus circunstancias.

En Argentina también existen nutridos antecedentes, dentro de los cuales sobresalen los estudios de Llach (Llach, 2006; Llach y Schumacher, 2003), Cervini (2002a, 2002b, 2003a, 2003b, 2004) y Cervini y Basualdo (2003). Llach (2006) se concentra en los factores escolares, su eficacia en la producción de resultados educativos y su distribución social. Utilizando distintas fuentes de información, crea múltiples indicadores del capital físico, capital humano y capital social de las escuelas y analiza su correlación con los resultados escolares. Concluye que los recursos escolares no son inocuos para la educación, sino que explican parcialmente su variabilidad, aún después de controlar por el nivel socioeconómico de los alumnos. Sin embargo, podría considerarse que este control es insuficiente dado que solo se emplea una estimación del consumo familiar per cápita, omitiendo otras dimensiones del acervo familiar que podrían estar correlacionadas con las variables dependientes e independientes. 
La investigación de Cervini (2002a) se concentra en los efectos de los recursos familiares sobre el rendimiento académico. Además, utiliza la técnica de regresión lineal por niveles múltiples que internaliza el hecho de que existen factores que afectan el resultado en diferentes instancias jerárquicamente ordenadas. Y, más importante aún, ofrece un marco teórico integral y coherente desde el cuál deducir el modelo a contrastar e interpretar los resultados, el de la reproducción cultural. Así lo describe el autor:

\begin{abstract}
La estrecha asociación entre origen social del alumno y su éxito o fracaso escolar es una manifestación empirica del proceso de reproducción de la desigualdad social. La teoría de la reproducción cultural formulada originalmente por Bourdieu [...] pretende explicar las desigualdades a través de una compleja trama de interacciones entre los condicionantes económicos y culturales y las prácticas del sistema educativo. Mediante la socialización familiar, el niño hereda cierto capital cultural acorde con su pertenencia de clase.
\end{abstract}

El capital cultural socialmente más valorado (dominante) es más probable que aparezca entre los núcleos de mayor nivel socioeconómico (background familiar); al mismo tiempo, la escuela tiende a valorar precisamente ese capital. Entonces, el niño de origen social alto tiene mayor probabilidad de éxito porque posee cierto capital cultural, heredado de sus padres y valorado por la escuela, que le ayuda a dominar el currículo escolar, a diferencia del procedente de familia con menor estatus social. El capital cultural juega, entonces, un papel de factor intermediario entre el .origen social del alumno (background familiar) y su aprendizaje. (p. 448)

Cervini encontró que el capital económico y cultural se asocia significativamente al resultado escolar, pero que no obstante ello, una proporción importante de la variación en el desempeño académico no es explicado por estos factores. Ello implicaría que las características escolares también influyen significativamente en el rendimiento.

En el ámbito de investigación para estudiantes universitarios de Argentina, los estudios realizados por Porto y Di Gresia (2001), Di Gresia, Porto y Ripani (2002), Fazio (2004) y Said Rücker, Chiapello y Espíndola (2009) abordan la temática desde un punto de vista similar al del presente trabajo y aportan valiosas referencias para efectuar un contraste de resultados. Por su parte, García (2014) sintetiza los estudios sobre rendimiento académico de los universitarios argentinos que se elaboraron en el periodo 2002-2012.

Con respecto a la conceptualización del nivel socioeconómico (NSE), Garbanzo Vargas (2013) destaca que el mismo comprende una serie de características del hogar asociadas con la capacidad de consumo del grupo familiar y que no debe confundirse o identificarse directamente con la pertenencia a una clase social determinada. Además, incluye igualmente rasgos cuantitativos y cualitativos, por lo que no resulta conveniente su medición en forma simple o lineal, ni puede pensarse como variable homogénea para distintos países. A pesar de ello, puede ser muy útil como instrumento de análisis a partir de la creación de grupos o categorías basadas en variables relacionadas con el consumo familiar.

Porto y Di Gresia (2001) estudian las características socioeconómicas de los estudiantes de una Facultad nacional (Cs. Económicas en la Universidad de la Plata). A partir de una encuesta realizada al efecto en el año 1999, encuentran que la cantidad de años de educación de los padres influye de manera positiva, mientras que el sexo masculino, la edad y las horas de trabajo lo hacen de manera negativa.

(...) son variables explicativas significativas del rendimiento estudiantil la carrera que cursa el estudiante, el sexo, la edad, la educación de los padres, la cantidad de horas que trabaja y las regulaciones de la Facultad de la condición de alumno regular. El tipo de escuela 
secundaria (pública-privada) y el lugar de procedencia del estudiante no tienen efecto significativo sobre el rendimiento [a partir de un análisis econométrico]. (p. 22)

En Di Gresia et al. (2002), la investigación se realiza sobre toda la población de universidades públicas a partir de los datos provistos por el Censo de Estudiantes de Universidades Nacionales del año 1994. Los resultados que presentan mayor interés para el presente trabajo son los siguientes:

[a] los varones tienen menor rendimiento en los estudios que las mujeres (...).

[b] El estado civil es un factor explicativo estadísticamente significativo.

[c] La escuela secundaria es un factor explicativo estadísticamente significativo del rendimiento: los alumnos que provienen de escuelas privadas rinden 0.10 materias más por año que los de escuelas públicas.

[d] Si el alumno cambia de residencia (se muda) para poder asistir a la universidad, ese cambio tiene un efecto positivo sobre el rendimiento. En cambio es negativo el impacto de tener que viajar entre distintas jurisdicciones (municipalidades) para asistir a la Facultad.

[e] Los años de permanencia en la Facultad tienen impacto negativo.

[f] Los estudiantes que dedican más horas al estudio, incluyendo clases, tienen mayor rendimiento.

[g]También es mayor el rendimiento cuanto mayor es la educación del padre y de la madre.

[h] Los estudiantes que trabajan tienen mayor rendimiento.

[i] Como era de esperar, el origen del financiamiento es una variable estadísticamente significativa: el mayor rendimiento corresponde a los estudiantes que se financian con beca y aporte familiar, luego los que tienen sólo beca de estudio, luego trabajo personal y aporte familiar y finalmente aporte familiar. En todos los casos, el resultado surge de la comparación con el financiamiento originado en el trabajo personal.

[...] En cuanto a las variables representativas (en forma indirecta) de calidad para cada universidad no se han encontrado relaciones estadísticamente significativas con el rendimiento. El $R^{2}$ de la regresión es relativamente bajo (0.12). (p. 32)

Fazio (2004) utiliza la misma base de datos del trabajo anterior, centrando su atención en la relación entre la situación laboral de los estudiantes y su rendimiento académico. Subraya que "más del 55\% de los estudiantes universitarios no se dedican exclusivamente a estudiar, sino que además trabajan. También es significativo el porcentaje que trabaja más de 35 horas semanales (24\%)" (p. 3).

En la justificación de la importancia de su estudio, la autora explica con notable lucidez que el vínculo situación laboral-rendimiento académico "tiene relevancia práctica por sus conclusiones acerca de las condiciones que debería reunir una experiencia laboral para preservar el desempeño académico del alumno (...)” (Fazio, 2004, p. 4). De acuerdo a sus resultados,

a) La incidencia positiva del trabajo del estudiante se manifiesta sólo cuando el trabajo implica una limitada dedicación horaria y un cierto grado de vinculación con los temas de la carrera que estudia.

b) Los trabajos sin vinculación con los temas de estudio afectan negativamente el rendimiento académico del alumno, cualquiera sea el nivel de horas trabajadas.

c) Un alumno que trabaje en temas vinculados con la carrera puede aumentar su rendimiento trabajando una moderada cantidad de horas semanales (hasta 5 horas diarias). (p. 26)

Said Rücker et al (2009) concluyen que las condiciones económicas desfavorables de los alumnos afectarían sustancialmente su rendimiento académico, al punto tal que 
directamente podrían impedir el desarrollo de sus estudios. Estos autores toman como indicador de situación socioeconómica adversa al índice de necesidades básicas insatisfechas (NBI) y al hecho de que el alumno se encuentre trabajando durante el cursado, en una investigación que reviste particular importancia porque también se enfoca en alumnos de la Universidad Nacional del Nordeste (UNNE).

En resumen, los autores citados en esta sección sostienen que las características socioeconómicas de los estudiantes son altamente significativas para explicar las diferencias en sus rendimientos académicos, aunque la incidencia de estos factores no es homogénea en todos los contextos (países y regiones).

También existe consenso en dejar de lado la visión pesimista inicial respecto de la eficacia de las variables escolares, a las que se les reconoce influencia en el desempeño académico, en especial, en países en desarrollo como los latinoamericanos (Cervini, Dari, Quiroz y Atorresi, 2014). Con esta base, cobra sentido la tarea de identificar la matriz de determinantes socioeconómicas del rendimiento escolar que es propia de una región y de un nivel educativo, con la finalidad de diseñar especialmente políticas educativas adecuadas al caso.

Finalmente, en cuanto a las posibles interpretaciones del concepto de Rendimiento Académico (RA), que es la variable explicada en el estudio, Montes Gutiérrez y Lerner Matiz (2010) sostienen que éste puede ser entendido de tres maneras:

a) como un resultado expresado e interpretado cuantitativamente; b) como juicio evaluativo (...) sobre (...) el proceso llevado a cabo por el estudiante; o c) de manera combinada asumiendo el rendimiento como proceso y resultado, evidenciado tanto en las calificaciones numéricas como en los juicios de valor sobre las capacidades y el 'saber hacer' del estudiante (...). (p. 12)

Aquí lo emplearemos en el primero de los sentidos, que es concordante también con la postura de Tosconi (2010). La autora define al RA como:

El nivel demostrado de conocimientos en un área o materia, evidenciado a través de indicadores cuantitativos, usualmente expresados mediante calificación ponderada en el sistema vigesimal y bajo el supuesto que es un "grupo social calificado" el que fija los rangos de aprobación, para áreas de conocimiento determinadas, para contenidos específicos o para asignaturas. (citado por Montes Gutiérrez y Lerner Matiz, 2010, p. 12)

En la misma línea, Edel Navarro (2003) remarca que "probablemente una de las variables más empleadas o consideradas por los docentes e investigadores para aproximarse al rendimiento académico son las calificaciones escolares"(p. 3); y siguiendo a Cascón (2000), afirma que:

(...) el indicador del nivel educativo adquirido, en este estado y en la práctica totalidad de los países desarrollados y en vías de desarrollo, ha sido, sigue y probablemente seguirán siendo las calificaciones escolares. A su vez, éstas son reflejo de las evaluaciones y/o exámenes donde el alumno ha de demostrar sus conocimientos sobre las distintas áreas o materias, que el sistema considera necesarias y suficientes para su desarrollo como miembro activo de la sociedad. (citado por Edel Navarro, 2003, p. 3)

Por tanto, siguiendo estas prácticas tradicionales en las investigaciones sobre eficacia escolar, asociaremos directamente las calificaciones de los alumnos con su rendimiento académico (aclarando que no es ésta la única manera de evaluar RA, aunque sí la utilizada comúnmente). 


\section{Método}

A continuación se expone el método empleado en la investigación, que incluye las fuentes de datos y muestreo, una descripción de las variables utilizadas y la estrategia de análisis.

\subsection{Fuente de datos y muestreo}

Los datos utilizados corresponden a dos fuentes: la Encuesta de Reinscripción (ER), contestada individualmente por los alumnos de distintas facultades de la Universidad Nacional del Nordeste (Argentina) en el año 2009, y los registros de calificaciones de alumnos de los Departamentos de Alumnado de las Facultades de Derecho y Odontología.

Estos fueron los mejores datos disponibles (por actualidad, universalidad y accesibilidad) al momento de llevar adelante el análisis. La demora en la sistematización de los datos recolectados anualmente y cierta reticencia en revelar datos que consideran información sensible del alumno (como sus calificaciones) han sido obstáculos para la obtención de una muestra de mayor extensión.

En la ER se colectan datos personales, de residencia, familiares, de situación laboral, de nivel educativo, de cobertura médica, de situación económica, entre otros; y es un requisito obligatorio para la reinscripción anual del alumno. No se consideran aquellos alumnos que, al momento de recabarse la información, estaban cursando su primer año de estudios. La cantidad de alumnos relevados por facultad se describe en la tabla 1.

La cantidad de alumnos con datos disponibles para el análisis corresponde a las Facultades de Derecho y Odontología con un total de 9.694 valores. No se disponen de datos de las restantes facultades.

\subsection{Descripción de las variables utilizadas}

El objetivo de este trabajo en el plano empírico es identificar las relaciones significativas entre el rendimiento académico del alumno, por un lado, y su nivel socioeconómico (NSE), por el otro. Si bien originariamente la idea de investigación incluía también el estudio del efecto de las variables académicas en el rendimiento, las severas dificultades experimentadas para la recolección de los datos necesarios obligó a dejar de lado, momentáneamente, dicha línea de análisis

La base de datos disponible no ofrece los indicadores ideales para evaluar las hipótesis que se plantean en esta investigación. Sin embargo, se disponen de datos que pueden emplearse como proxies para inferir algunas relaciones (Anexo 1).

Tabla 1. Cantidad de estudiantes matriculados por Facultad o Instituto. UNNE

\begin{tabular}{lcc}
\hline \multicolumn{1}{c}{ UNIDAD ACADÉMICA } & CANTIDAD DE ALUMNOS & \% DEL TOTAL \\
\hline Fac.de Derecho, Cs. Soc. y Políticas & 9.059 & $34 \%$ \\
Fac. de Cs. Exactas y Naturales y Agrimensura & 4.855 & $18 \%$ \\
Fac. de Humanidades & 3.270 & $12 \%$ \\
Fac. de Arquitectura y Urbanismo & 2.420 & $9 \%$ \\
Fac. de Cs. Veterinarias & 1.875 & $7 \%$ \\
Fac. de Ciencias Agrarias & 1.334 & $5 \%$ \\
Fac. de Odontología & 1.297 & $5 \%$ \\
Instituto de Criminalística & 922 & $4 \%$ \\
Fac. de Ingeniería & 1.053 & $4 \%$ \\
Inst. de Adm. de Empresas Agropecuarias & 174 & $1 \%$ \\
Total general & 26.259 & $100 \%$ \\
\hline Fuente. Elaboración propia. & &
\end{tabular}


Con respecto al RA, se dispone del promedio histórico, que es el promedio de todas las calificaciones obtenidas por el alumno, incluyendo aplazos. Esta será la variable a explicar a lo largo del análisis. Es importante aclarar que en el conjunto de datos se incluyen alumnos con distintos grados de avance en las carreras. También corresponde explicitar que la investigación supone que las notas en distintas carreras y facultades son comparables en forma directa.

Las variables explicativas utilizadas en el análisis pueden agruparse en clases o categorías:

- Variables de Perfil General: unidad académica a la que pertenece el alumno, sexo, edad y necesidad de traslado o desplazamiento (variable categórica que describe si el alumno es o no originario de la ciudad donde cursa sus estudios).

- Variables de Familia Núcleo: describen el estado civil del alumno, la cantidad de hijos, y de familiares que están a su cargo.

- Variables de Perfil Laboral: se refieren a la inserción del alumno en el mercado laboral, incluida su condición de ocupación, tipo de ocupación, si se le descuentan aportes jubilatorios (es decir, si está formalmente registrado como trabajador ante los organismos de la seguridad social), horas de trabajo y relación del trabajo con los estudios.

- Variables de Nivel Socioeconómico (NSE): se agrupan aquí los indicadores de la base de datos que se consideraron proxies relevantes de nivel socioeconómico. La primera de ellas es la cobertura médica. Se trabaja bajo el supuesto de que ésta se relaciona razonablemente con el NSE. Así, quienes poseen cobertura médica tendrían un NSE superior a quienes no la poseen. A su vez, dentro de quienes cuentan con este beneficio, las obras sociales prepagas (privadas) o por afiliación voluntaria denotan un NSE aún mayor. La segunda variable proxy es la forma de financiamiento de los estudios. En particular, la financiación de los estudios a través de planes o programas sociales denota un menor NSE. Finalmente, se analiza la condición de beneficiario de becas. Las mismas se otorgan generalmente a quienes pueden demostrar un bajo NSE, por lo que se usará esta variable en ese sentido.

- Variables de Familia de Origen: describen la condición laboral y el nivel de estudio de los padres. Estas variables son utilizadas como indicadores del entorno sociocultural de origen del alumno

\subsection{Estrategia de análisis}

En primer lugar se realiza una caracterización general de la muestra. Luego se describen los datos de rendimiento académico para las unidades de las que se disponen datos suficientes, que son las Facultades de Derecho y Odontología.

Finalmente se estiman las correlaciones entre el rendimiento académico y las variables de perfil empleando regresiones múltiples por Mínimos Cuadrados Ordinarios (MCO). Cervini (2002a) observa que en este tipo de investigaciones es preferible recurrir a los modelos jerárquicos lineales antes que al método de MCO. Sin embargo, los datos disponibles no son suficientes para la aplicación del modelo sugerido por este autor (la única agrupación jerárquica con la que podemos contar es la que separa a los alumnos de Derecho y a los de Odontología). Pese a esta limitación, se realizan regresiones separadas 
para cada Facultad en el Anexo 3 con el objetivo de identificar posibles diferencias estructurales en los efectos.

Este método empleado (MCO) nos permite estimar el efecto independiente o ceteris paribus de cada variable explicativa en el RA. Por ejemplo, es esperable que las variables Situación Laboral y Obra Social estén correlacionadas, ya que estar empleado aumenta las probabilidades de tener obra social por el propio trabajo (la correlación no es exacta por la existencia del trabajo no registrado o informal). Si analizamos por separado el efecto de estas variables sobre RA es posible que lo sobreestimemos o subestimemos, ya que, al covariar, cada variable explicativa esconderá el efecto de la otra. Con la regresión múltiple por MCO podemos aislar el efecto individual de cada una manteniendo a la otra constante (ceteris paribus)

Se estima el siguiente modelo:

$$
R A=\beta_{0}+\beta_{1} X_{1}+\cdots+\beta_{n} X_{n}+u
$$

donde $R A$ es el rendimiento académico, $X_{1}, \ldots, X_{n}$ son las variables explicativas y $u$ ues el error que incorpora el efecto de todas las demás variables no incluidas en el modelo. El coeficiente $\beta_{i}$ uede entenderse como el efecto independiente (ceteris paribus) de un aumento unitario de la variable $X_{i}$ en el RA. El supuesto central que se efectúa al utilizar este método es que no hay variables omitidas que estén correlacionadas con los regresores. Por ejemplo, si existe la variable "Inteligencia individual" que no está incluida en el modelo pero está correlacionada con la Situación Laboral del alumno, el $\beta_{i}$ estimado de esta última estará sesgado. Wooldridge (2002) ofrece mayores detalles sobre los supuestos implicados en la regresión múltiple por MCO.

En esta instancia se corren cuatro modelos incorporando las variables explicativas de a una a la vez, con el propósito de identificar su efecto marginal:

- General (Modelo 1): incluye todos los datos, sin distinción de unidades académicas. Se intenta maximizar el número de observaciones válidas, dejando de lado variables con una elevada proporción de valores perdidos.

- Por género (Modelo 2): se compara el comportamiento de las estimaciones entre los subconjuntos de hombres y mujeres para identificar patrones de correlación con perspectivas de género.

- Subconjunto Ocupados (Modelo 3): solo se incluyen los alumnos ocupados para identificar su impacto en el rendimiento.

- Subconjunto Padres (Modelo 4): se incorporan las variables referentes a la ocupación y el nivel de educación de los padres para estimar el efecto del entorno sociocultural de la familia de origen en el RA (solo se dispone de datos de la Facultad de Odontología).

- Por unidad académica (Modelo en Anexo 3): se compara el comportamiento de las estimaciones entre las facultades para identificar efectos estructuralmente distintos entre ambas y probar la robustez de los resultados. 


\section{Resultados}

\subsection{Descripción general de la muestra}

El 54\% de la población son estudiantes mujeres. La edad promedio es de 27 años con desvío estándar de 7.6 años y mediana de 25 años. El 42\% de los alumnos estudia en la misma localidad de su residencia habitual. Un $87 \%$ del alumnado es soltero, y sólo uno de cada cuatro alumnos tiene hijos (60\% de los cuales posee un único hijo).

Uno de cada tres alumnos trabaja mientras cursa sus estudios universitarios, proporción que es menor a la hallada en investigaciones análogas como la de Fazio (2004), quien encontró que la proporción de estudiantes que trabajan y estudian fue del $55 \%$ para todo el país en el año 1994, y la Porto (2001), donde se halló que el 45\% de los estudiantes trabajaban en forma simultánea al cursado de sus carreras (en el año 1999, en una Facultad de la Universidad de la Plata).

Aquí uno de cada siete estudiantes busca trabajo pero no lo encuentra. El resto se encuentra fuera del mercado laboral. Entre los ocupados, predomina la condición de empleado (60\%), y un $28.5 \%$ realiza tareas estrechamente vinculadas a sus estudios universitarios. El 8.64\% reviste la calidad de pasante.

Se destaca que un $47.5 \%$ no tiene cobertura de salud. Entre quienes sí poseen cobertura, más de la mitad la obtuvo por estar a cargo de un titular que cuenta con el beneficio. Un $11 \%$ de los alumnos tiene obra social en razón de su propio trabajo y el $4 \%$ corresponde a afiliados voluntarios.

Solo $40 \%$ de los alumnos que trabajan manifiesta estar efectuando aportes jubilatorios. Este hecho, sumado a que solamente un tercio de los que tienen trabajo poseen obra social, puede ser considerado como indicador del nivel de precariedad e informalidad laboral de los alumnos.

Las contribuciones económicas familiares constituyen la principal fuente de financiamiento de los estudios ( $70 \%$ de los alumnos). El financiamiento a través de becas, planes sociales u otras fuentes son mucho menos relevantes en la muestra. De los alumnos becados, más de la mitad reciben beneficios de la universidad (principalmente becas de tipo económico), mientras que un $37 \%$ son beneficiarios de becas otorgadas por el Estado nacional.

Respecto al nivel de educación alcanzado por los padres, predominan quienes no han completado los estudios secundarios. Sin embargo, los padres que han finalizado estudios universitarios son un grupo importante: la UNNE duplica la proporción de alumnos con madres con formación universitaria que, por ejemplo, la Universidad Nacional de La Plata (véase Porto y Di Gresia, 2001). Es importante destacar que solo el 61,2\% de los alumnos respondió las preguntas acerca del nivel educativo de sus padres.

En cuanto a la situación laboral de los padres, predomina la característica de ocupado, con mayor proporción de padres ocupados, y en su mayoría corresponde a la categoría de empleados (seguidos por los cuentrapropistas).

\subsection{Rendimientos académicos en Derecho y Odontología}

La media general de la variable "rendimiento académico" de los alumnos de la muestra es de 6.78 puntos, mientras que la mediana es ligeramente superior (tabla 2). El desvío típico encontrado es de 0.84 , lo que denota una fuerte concentración de las calificaciones 
alrededor del indicador de tendencia central. Prueba de ello es que el $77 \%$ de los alumnos tiene entre 6 y 8 de promedio.

Tabla 2. Estadísticas descriptivas del rendimiento académico. UNNE

\begin{tabular}{lrcccc}
\hline & MEDIA & DESV. TÍP. & MEDIANA & RANGO & ASIMETRÍA \\
\hline Facultad de Derecho & 6.74 & 0.84 & 6.8 & 10.0 & -0.6 \\
Facultad de Odontología & 7.24 & 0.84 & 7.2 & 4.5 & 0.2 \\
Total & 6.78 & 0.85 & 6.8 & 10.0 & -0.5 \\
\hline
\end{tabular}

Fuente: Elaboración propia.

Existen diferencias entre unidades académicas y entre carreras. Mientras que el promedio en Abogacía (que es una de las carreras que se estudian en la Facultad de Derecho, las otras son Notariado y Procuración) es de 6.3 puntos, en la carrera de Odontología es de 7.24 puntos, un $7.6 \%$ superior. La dispersión entre carreras no es significativamente diferente. Por otro lado, mientras en Derecho la asimetría de la distribución se produce hacia la izquierda (promedios bajos), en Odontología se produce hacia la derecha (promedios altos).

\subsection{Relación entre NSE y RA. Modelo 1}

El eje central del trabajo consiste en estimar el impacto del NSE del alumno en el rendimiento académico. En esta sección se pretende aproximar empíricamente esta relación a partir de los datos disponibles. En el Anexo 2 puede verse la cantidad de observaciones perdidas por falta de respuesta en cada variable.

La tabla 3 presenta los resultados obtenidos al regresar las variables sobre el promedio académico. En cada paso se incluyó un grupo de variables, con la excepción de los indicadores de NSE, que se incluyeron de uno en uno. Se presentan los coeficientes estimados, los coeficientes beta y el grado de significancia. Estos coeficientes beta son estimaciones con variables estandarizadas: al normalizar los argumentos de una regresión, el rango de variación es idéntico para todos ellos, de manera que podemos comparar sus efectos relativos directamente (Wooldridge, 2002).

El Modelo 1a regresa sexo y edad sobre la variable dependiente: se verifica que las mujeres tienen un RA superior a los hombres, situación que se mantiene a medida que se ingresan más regresores.

No ocurre lo mismo con la edad. Esta se presenta estadísticamente no significativa en el Modelo 1a, aunque gana peso a medida que se incluyen otras variables. El efecto final no es lineal, sino que presenta una forma cuadrática. En concreto, la edad del alumno tiene un efecto positivo en el RA hasta aproximadamente los 28 años. De allí en adelante su efecto es negativo.

Dentro del grupo de variables "familia núcleo", las más significativas son estado civil casado y cantidad de hijos. Los estudiantes casados presentan un rendimiento sistemáticamente superior a los solteros. La cantidad de hijos afecta negativamente el rendimiento: cada hijo disminuye el promedio del alumno (padre/madre) en alrededor de 0.10 puntos. Ambos efectos son robustos a la introducción de nuevas variables.

Con respecto a la situación laboral, se verifica que tanto si está ocupado como si está desocupado (es decir, no tiene trabajo pero lo busca activamente), el alumno presenta un rendimiento académico inferior al del grupo base (que no tiene interés ni intención de ingresar al mercado laboral). 
Tabla 3. Modelo 1. Estimaciones generales. UNNE

\begin{tabular}{|c|c|c|c|c|c|c|c|c|c|c|c|c|c|c|c|c|c|c|}
\hline & \multicolumn{3}{|c|}{ MOD. 1A } & \multicolumn{3}{|c|}{ MOD. 1B } & \multicolumn{3}{|c|}{ MOD. 1C } & \multicolumn{3}{|c|}{ MOD. 1D } & \multicolumn{3}{|c|}{ MOD. 1E } & \multicolumn{3}{|c|}{ MOD. 1} \\
\hline & B & Beta & Sig. & B & Beta & Sig. & B & Beta & Sig. & B & Beta & Sig. & B & Beta & Sig. & B & Beta & Sig. \\
\hline (Constante) & 6,40 & & ***** & 6,37 & & **** & 6,06 & & ***** & 6,01 & & **** & 5,82 & & **** & 5,82 & & ****** \\
\hline Sexo & 0,21 & 0,13 & $* * *$ & 0,21 & 0,13 & $* * *$ & 0,20 & 0,12 & $* * *$ & 0,20 & 0,12 & $* * *$ & 0,20 & 0,11 & $* * *$ & 0,20 & 0,11 & ***** \\
\hline Edad_est & 0,00 & 0,02 & & 0,00 & 0,03 & & 0,03 & 0,30 & $* * *$ & 0,03 & 0,29 & $* * *$ & 0,03 & 0,33 & $* * *$ & 0,03 & 0,33 & **** \\
\hline Edad_est_2 & 0,00 & 0,02 & & 0,00 & 0,03 & & 0,00 & $-0,18$ & *** & 0,00 & $-0,18$ & $* *$ & $\mathrm{OE}, \mathrm{OO}$ & $-0,20$ & $* * *$ & 0,00 & $-0,20$ & **** \\
\hline EC_casado & & & & 0,15 & 0,06 & $* * *$ & 0,15 & 0,06 & $* * *$ & 0,14 & 0,06 & $* * *$ & 0,15 & 0,06 & $* * *$ & 0,15 & 0,06 & $* * *$ \\
\hline EC_otros & & & & 0,02 & 0,01 & & 0,03 & 0,01 & & 0,02 & 0,01 & & 0,04 & 0,01 & & 0,04 & 0,01 & \\
\hline cant_hijos_alum & & & & $-0,08$ & $-0,09$ & $* * *$ & $-0,08$ & $-0,08$ & $* * *$ & $-0,08$ & $-0,09$ & $* * *$ & $-0,08$ & $-0,08$ & **** & $-0,08$ & $-0,08$ & **** \\
\hline sit_lab_ocup & & & & & & & $-0,24$ & $-0,14$ & $* * *$ & $-0,25$ & $-0,15$ & $* * *$ & $-0,21$ & $-0,12$ & **** & $-0,21$ & $-0,12$ & **** \\
\hline sit_lab_desocup & & & & & & & $-0,23$ & $-0,10$ & **** & $-0,23$ & $-0,10$ & **** & $-0,23$ & $-0,10$ & **** & $-0,23$ & $-0,10$ & **** \\
\hline OS_fam & & & & & & & & & & 0,07 & 0,04 & **** & 0,07 & 0,04 & **** & 0,07 & 0,04 & **** \\
\hline OS_trab & & & & & & & & & & 0,11 & 0,05 & **** & 0,14 & 0,06 & $* * *$ & 0,14 & 0,06 & **** \\
\hline OS_afil & & & & & & & & & & 0,18 & 0,05 & **** & 0,18 & 0,05 & $* * *$ & 0,18 & 0,06 & **** \\
\hline OS_uni & & & & & & & & & & 0,19 & 0,05 & **** & 0,18 & 0,05 & $* * *$ & 0,18 & 0,05 & **** \\
\hline alu_cos_est_ap_fam & & & & & & & & & & & & & 0,13 & 0,07 & **** & 0,13 & 0,07 & **** \\
\hline alu_cos_est_trab & & & & & & & & & & & & & 0,03 & 0,02 & & 0,03 & 0,02 & \\
\hline alu_cos_est_beca & & & & & & & & & & & & & 0,36 & 0,05 & $* * *$ & 0,14 & 0,02 & \\
\hline alu_cos_est_plsoc & & & & & & & & & & & & & $-0,30$ & $-0,03$ & $* * *$ & $-0,30$ & $-0,03$ & **** \\
\hline alu_cos_est_otra & & & & & & & & & & & & & $-0,06$ & $-0,01$ & & $-0,06$ & $-0,01$ & \\
\hline tiene_beca & & & & & & & & & & & & & & & & 0,24 & 0,03 & \\
\hline cant_becas_uni & & & & & & & & & & & & & & & & $-0,01$ & 0,00 & \\
\hline $\mathrm{R}^{2}$ corregido & & 0,02 & & & 0,02 & & & 0,03 & & & 0,04 & & & 0,04 & & & 0,04 & \\
\hline $\mathrm{F}$ & & 42,04 & & & 26,35 & & & 34,63 & & & 26,54 & & & 21,33 & & & 19,10 & \\
\hline Observaciones & & $7514, \mathrm{OO}$ & & & 7514,00 & & & 514,00 & & & $7514, \mathrm{OO}$ & & & 7514,00 & & & $7514, \mathrm{OO}$ & \\
\hline
\end{tabular}

Nota: se presentan los coeficientes beta (variables estandarizadas) de la regresión por Mínimos Cuadrados Ordinarios. La variable dependiente es el "Rendimiento

Académico". Las variables independientes fueron introduciéndose en 6 etapas: 1) sexo y edad, 2) estado civil y cantidad de hijos, 3) situación laboral del alumno, 4) cobertura de obra social del alumno, 5) forma de costeo de estudios, 6) posesión y cantidad de becas. ${ }^{*} \mathrm{p}<0,10$; ** p $<0,05$; *** p $<0,01$. Fuente: Elaboración propia. 
Uno de los indicadores más confiables de NSE es el tipo de cobertura médica del alumno, que está asociado a un mayor RA en comparación con el grupo que no posee cobertura. Habitualmente, contarán con cobertura las siguientes personas: aquellas que cuenten con un familiar (padres o cónyuges) que trabaje en relación de dependencia formalmente registrada, aquellos que trabajen en relación de dependencia formalmente registrada, aquellos trabajadores (autónomos) que contraten un servicio privado de obra social, aquellos alumnos que se inscriban en el régimen de servicios sociales administrado por la Universidad. Los principales casos de alumnos sin cobertura comprenden: aquellos cuyos padres no poseen trabajo o no tienen trabajo formal, y aquellos alumnos que trabajan en relaciones laborales no registradas.

Dentro del grupo con cobertura, quienes tienen obra social por ser familiares a cargo del beneficiario directo presentan el menor coeficiente que aquellos que la tienen por su propio trabajo. Los que tienen obra social por afiliación voluntaria o provista por la Universidad son quienes tienen aún mayor diferencia de rendimiento. Este es un efecto adicional a la situación laboral que indica no solo que está ocupado, sino también que aporta a una obra social (ver Modelo 1d).

Otro de los indicadores de NSE es la fuente de financiamiento de los estudios (recuérdese que esta es una pregunta de respuesta múltiple, por lo que las opciones no son excluyentes. Por ejemplo, un alumno puede recibir aportes familiares y una beca al mismo tiempo). Quienes reciben aportes familiares tienen un rendimiento sistemáticamente superior a quienes no los reciben. El financiamiento con recursos derivados del propio trabajo no tiene un efecto adicional a las variables laborales ya consideradas, mientras que el financiamiento por becas tiene un efecto fuertemente positivo.

En el modelo 1 se incluyen variables adicionales sobre becas. Sin embargo, su efecto no es significativo (al medir fenómenos tan similares, se quita significatividad estadística a la variable de financiamiento de estudios). Quienes se financian con planes sociales tienen un rendimiento fuertemente inferior a quienes no usan esta fuente. Es necesario destacar que, a pesar de que la significatividad de los efectos encontrados es importante, el modelo final no explica más que el $4 \%$ de la variabilidad de los rendimientos. Este valor guarda similitud con los encontrados en investigaciones análogas de rendimiento educativo, como las de Porto (2001), Di Gresia et al. (2002). No obstante, el modelo en su conjunto es altamente significativo.

Con el objetivo de encontrar diferencias en el impacto de las variables socioeconómicas entre Facultades, se corrió el modelo por separado para cada una de ellas (ver Anexo 3). En Odontología las variables edad, estado civil, obra social por afiliación voluntaria y costeo de estudio por planes sociales se presentan como significativos, siendo ésta última la que mayor impacto (negativo) produce en el RA. La baja significatividad estadística de las demás variables puede explicarse por el menor tamaño de la muestra. En Derecho, la mayor parte de los resultados hallados en el apartado anterior se conservan.

\subsection{Diferencia de género. Modelo 2}

El estudio del impacto diferencial de las variables socioeconómicas de acuerdo al género del alumno es fundamental para analizar si las oportunidades educativas se distribuyen igualitariamente. A continuación se evalúan los efectos del modelo 1 en hombres y mujeres por separado (tabla 4 ). 
Tabla 4. Modelo 2. Comparación de resultados por género. UNNE

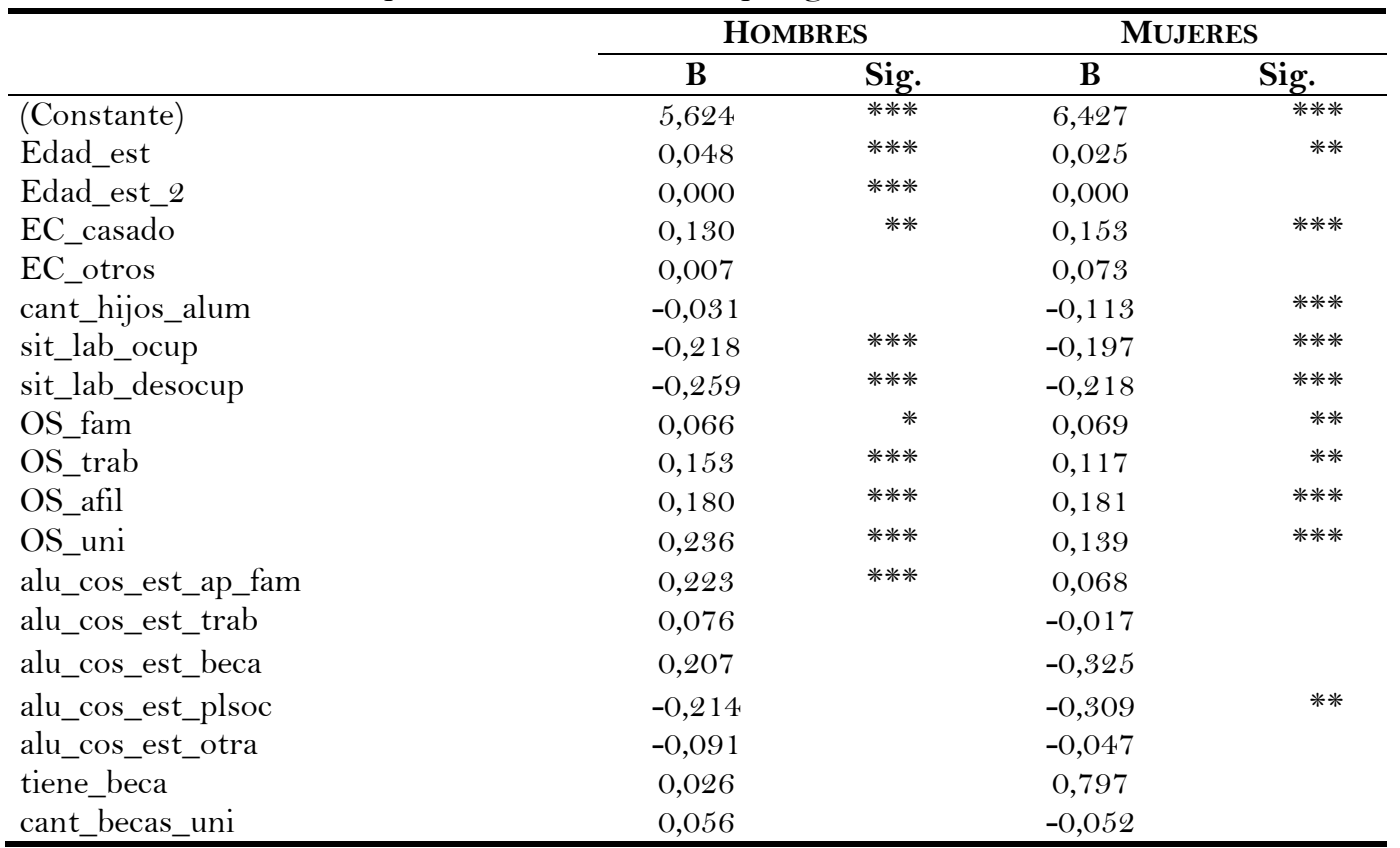

Nota: se presentan los coeficientes de la regresión por Mínimos Cuadrados Ordinarios. La variable dependiente es el "Rendimiento Académico". * $\mathrm{p}<0,10$. ** $\mathrm{p}<0,05$. *** $\mathrm{p}<0,01$.

Fuente: Elaboración propia.

Las diferencias más notables se verifican en la edad, cantidad de hijos, situación laboral desocupado, obra social universitaria y forma de costeo de estudios. En cuanto a la edad, en el grupo de hombres se mantiene la relación descripta anteriormente. En las mujeres, en cambio, la relación es lineal y directa: a mayor edad, mejor RA. La diferencia más destacable en materia de género es el efecto de la cantidad de hijos del alumno, que no afecta el rendimiento del alumno de sexo masculino pero sí lo hace y de manera intensa y negativa cuando se trata del sexo femenino. Finalmente, los aportes económicos de origen familiar son estadísticamente significativos para explicar el RA de los hombres pero no el de las mujeres. Lo contrario ocurre con los planes sociales.

\subsection{Perfil laboral. Modelo 3}

En esta sección se profundiza en el efecto de las variables laborales sobre el RA con un modelo ampliado (tabla 5). El subconjunto utilizado para la regresión es el de los alumnos ocupados.

Como resultados destacados, se verifica un mayor rendimiento de los ocupados cuentapropistas por sobre los ocupados empleados, y la cantidad de horas trabajadas se relaciona positivamente con el RA, al contrario de lo hallado en otros estudios (como el citado de Fazio, 2004). Finalmente, y de manera notable, el hecho de que el trabajo desempeñado tenga relación con el campo de estudios del alumno tiene un efecto positivo en su rendimiento. Fazio (2004) encontró este mismo vínculo, agregando que el efecto positivo se mantiene únicamente hasta cierta cantidad de horas semanales trabajadas, luego de lo cual el trabajo comienza a incidir negativamente en el rendimiento aunque se desarrolle en un área vinculada al de los estudios. 
Tabla 5. Modelo 3. Efectos de las características del empleo. UnNE

\begin{tabular}{|c|c|c|c|}
\hline VARIABLES & $\bar{B}$ & BETA & SIG. \\
\hline (Constante) & 5,76 & & **** \\
\hline Sexo & 0,17 & 0,10 & **** \\
\hline Edad_est & 0,04 & 0,39 & **** \\
\hline Edad_est_2 & 0,00 & $-0,25$ & ** \\
\hline EC_casado & 0,18 & 0,09 & **** \\
\hline EC_otros & 0,13 & 0,04 & ** \\
\hline cant_hijos_alum & $-0,08$ & $-0,10$ & **** \\
\hline OS_fam & 0,10 & 0,04 & ** \\
\hline OS_trab & 0,14 & 0,08 & **** \\
\hline OS_afil & 0,23 & 0,07 & **** \\
\hline OS_uni & 0,20 & 0,05 & **** \\
\hline alu_cos_est_ap_fam & 0,15 & 0,08 & **** \\
\hline alu_cos_est_trab & 0,07 & 0,03 & \\
\hline alu_cos_est_beca & 0,02 & 0,00 & \\
\hline alu_cos_est_plsoc & $-0,14$ & $-0,01$ & \\
\hline alu_cos_est_otra & 0,01 & 0,00 & \\
\hline tiene_beca & 0,32 & 0,04 & \\
\hline cant_becas_uni & $-0,03$ & $-0,01$ & \\
\hline sit_ocup_pat & 0,14 & 0,03 & \\
\hline sit_ocup_cta & 0,08 & 0,04 & ** \\
\hline sit_ocup_pas & 0,01 & 0,00 & \\
\hline alu_trab_desjub & $-0,06$ & $-0,04$ & \\
\hline hora_sem_trab_alum & 0,03 & 0,05 & ** \\
\hline rel_trab_carrera & 0,16 & $-0,16$ & **** \\
\hline $\mathrm{N}$ & 3107 & & \\
\hline $\mathrm{R}^{2}$ corregido & 0,07 & & \\
\hline
\end{tabular}

Nota: se presentan los coeficientes regulares (B) y beta (variables estandarizadas) de las regresiones por MCO para el sub-conjunto de los "Ocupados". La variable dependiente es el "Rendimiento Académico". Se agregan variables independientes vinculadas a las características de su empleo.

$* \mathrm{p}<0,10 . * * \mathrm{p}<0,05 . * * * \mathrm{p}<0,01$.

Fuente: Elaboración propia.

\subsection{Perfil de los padres. Modelo 4}

Se incorporan al modelo las variables referentes al nivel de educación y estatus laboral de los padres. Solo se utilizan datos referentes a la Facultad de Odontología (tabla 6). Esto puede explicar, en parte, la no significatividad de algunas variables por las razones expuestas previamente.

La única variable que presenta un efecto estadísticamente significativo es el máximo nivel educativo alcanzado por los padres: cuanto mayor es la educación de los padres, mayor es el RA del alumno. Las variables de situación laboral de los padres no inciden en el RA.

\section{Discusión}

Los resultados hallados permiten abrir el debate en torno a numerosas cuestiones, las que no pueden ser agotadas en el acotado ámbito del presente artículo. Hecha la salvedad y sin perjuicio de las observaciones que serán vertidas en las conclusiones, proponemos enfatizar las siguientes:

Los promedios de calificaciones de los alumnos de la Facultad de Odontología son superiores a los de Derecho, y estas diferencias se mantienen luego de considerar muchas otras variables, incluidas algunas referentes al NSE del alumno. Esto puede deberse a 
diversas causas. Una posible explicación radica en la diferencia en la dotación de recursos académicos (planteles docentes, infraestructura, etc.) entre facultades. Desafortunadamente, la falta de datos respecto a los resultados académicos de las demás Facultades impide contrastar hipótesis como las siguientes:

- Las facultades que tienen más y mejor personal docente por alumno obtienen mejores RA.

- Las facultades que tienen recursos físicos más adecuados para las necesidades del aprendizaje en el campo de conocimiento propio tienen mejores RA.

- Las facultades que tienen una gestión más organizada y eficiente obtienen mejores RA.

Tabla 6. Modelo 4. Efecto de la educación y ocupación de los padres. UNNE

\begin{tabular}{|c|c|c|c|}
\hline VARIABLES & B & BETA & SIG. \\
\hline (Constante) & 9,19 & & **** \\
\hline Sexo & 0,05 & 0,03 & \\
\hline Edad_est & $-0,16$ & $-0,90$ & $* * *$ \\
\hline Edad_est_2 & 0,00 & 0,73 & ***** \\
\hline EC_casado & 0,21 & 0,05 & \\
\hline EC_otros & 0,58 & 0,06 & \\
\hline cant_hijos_alum & 0,02 & 0,01 & \\
\hline OS_fam & 0,08 & 0,05 & \\
\hline OS_trab & 0,21 & 0,03 & \\
\hline OS_afil & 0,19 & 0,05 & \\
\hline OS_uni & $-0,11$ & $-0,03$ & \\
\hline alu_cos_est_ap_fam & 0,09 & 0,02 & \\
\hline alu_cos_est_trab & $-0,02$ & $-0,01$ & \\
\hline alu_cos_est_plsoc & $-1,54$ & $-0,07$ & $*$ \\
\hline alu_cos_est_otra & 0,12 & 0,01 & \\
\hline tiene_beca & 0,17 & 0,03 & \\
\hline cant_becas_uni & 0,00 & 0,00 & \\
\hline max_est_padres & 0,16 & 0,13 & ***** \\
\hline sit_lab_pad_ocu & 0,06 & 0,02 & \\
\hline sit_lab_pad_des & 0,12 & 0,01 & \\
\hline sit_lab_mad_ocu & 0,05 & 0,03 & \\
\hline sit_lab_mad_des & 0,94 & 0,04 & \\
\hline $\mathrm{N}$ & 634 & & \\
\hline $\mathrm{R}^{2}$ corregido & 0,10 & & \\
\hline
\end{tabular}

Nota: se presentan los coeficientes regulares (B) y beta (variables estandarizadas) de las regresiones por MCO para el sub-conjunto de los alumnos que respondieron las preguntas referidas al nivel de estudios y situación laboral de sus padres. Fueron excluidos todos los alumnos de la Fac. de Derecho por no respuesta. La variable dependiente es el "Rendimiento Académico". Se agregan variables independientes vinculadas al nivel de estudio y situación laboral de los padres.

$* \mathrm{p}<0,10 . * * \mathrm{p}<0,05 . * * * \mathrm{p}<0,01$.

Fuente: Elaboración propia.

Además de estas hipótesis, podría testearse la significancia de muchas otras variables, como la cultura organizacional, el nivel de compromiso de los actores, el presupuesto, la dinámica científica del campo de conocimiento involucrado, entre otras. Otras posibles explicaciones podrían estar asociadas a la definición adoptada de RA, a la aptitud del promedio de calificaciones como instrumento central de medición del desempeño del alumno y a la razonabilidad de la comparación de promedios entre facultades o universidades. Ellas integran el marco de presupuestos de la presente investigación, en el 
cual se asume que es válido utilizar al promedio histórico como indicador del rendimiento académico y que las calificaciones obtenidas en las distintas unidades académicas son directamente comparables -Cascón (2000) ha analizado la factibilidad de la utilización de las calificaciones del alumno como predictor del RA-.

Parece evidente que la situación familiar del alumno afecta directamente su rendimiento. El estudio indica que el estado civil casado se asocia positivamente al RA, mientras que tener hijos a cargo se vincula con RA inferiores. Si bien es esperable y comprensible que el cuidado de los hijos demande horas y energías que no podrán destinarse al estudio (disminuyendo el RA), no es sencillo explicar por qué los alumnos casados tienen mejores calificaciones (es posible que cuenten con un mayor grado de madurez o que ello indique pertenencia a un NSE más alto).

Tanto si el alumno se halla trabajando como si se encuentra en la búsqueda de un puesto, su RA es significativamente inferior al de quienes estudian a tiempo completo (y no desean acceder al mercado laboral). Detrás de estas variables, pueden existir dos causas distintas. Por un lado, las diferencias en la cantidad de tiempo disponible para el estudio con que cuentan los alumnos en cada caso. Por otra parte, es probable que la situación laboral esté operando aquí como un indicador de NSE (quienes necesitan trabajar poseen un NSE menor a quienes pueden dedicarse exclusivamente al estudio).

El nivel de instrucción de los padres resulta significativo en la explicación el RA de sus hijos, no así su estado de ocupación actual. Esto es consistente con los argumentos teóricos mencionados previamente (T. de la Reproducción Cultural). También coincide con los antecedentes locales, en los cuáles se lo considera uno de los factores de mayor peso o incidencia en el RA (García, 2014). Queda pendiente la indagación en torno a las posibles diferencias de efecto según género del progenitor instruido.

$\mathrm{Al}$ interpretar los resultados obtenidos, así como al reflexionar sobre las implicancias de los mismos no puede soslayarse que la meta en este tipo de investigaciones es la detección de relaciones entre variables y la determinación del sentido e intensidad de las mismas. Las causas o razones subyacentes de las relaciones pasan, por este motivo, a un segundo plano. Por lo tanto, sin perjuicio de que puedan mencionarse posibles teorías explicativas o adelantarse conjeturas al respecto, el enfoque adoptado es eminentemente pragmático. Valga un ejemplo para reflejar esta afirmación: el hallazgo de una relación negativa significativa e intensa entre cantidad e hijos y RA constituye aquí un producto suficiente per se, con independencia del canal preciso a través del cual se produce el efecto (que puede a su vez ser punto de partida de otras investigaciones con características distintas).

\section{Conclusiones}

Del análisis descriptivo surge que el alumno modal es uno de 25 años, proveniente del interior de las provincias, soltero, sin hijos o familiares a cargo, estudiante a tiempo completo, que se financia a través de aportes familiares, con padres ocupados pero sin estudios secundarios completos.

Sin embargo, existe una fuerte dispersión a partir de este perfil, en particular en lo referente a indicadores de NSE. Hay tantos alumnos con cobertura médica como sin ella. Hay una notoria proporción (próxima al tercio) de alumnos que se encuentran trabajando al mismo tiempo que cursan sus estudios (gran parte de los cuales se encontrarían en 
situación laboral precaria o informal). Finalmente, hay una muy pequeña fracción del alumnado (cercano al $2 \%$ ) que recibe becas.

En relación con los objetivos del trabajo, se encontró evidencia contundente de que las variables de NSE son significativas para explicar las diferencias de rendimiento académico entre los alumnos de la UNNE. Las variables que se han utilizado como indicadores de NSE elevado han presentado una correlación positiva, y las variables que se emplearon como indicadores de NSE más bajo han presentado una correlación negativa.

En la tabla 7 se presenta un resumen de los efectos aquí encontrados y una comparación con otros trabajos realizados sobre el universo de estudiantes de universidades nacionales, en especial Di Gresia y colaboradores, (2002); Fazio (2004) y Said Rücker y colaboradores (2009).

En materia de género, el hallazgo más relevante se manifiesta en el desempeño de las estudiantes mujeres con hijos: su rendimiento es notoriamente inferior al de los estudiantes hombres con hijos. Esto parecería indicar que la responsabilidad del cuidado de los hijos repercute principalmente en el rendimiento académico de estudiantes de sexo femenino. Para revertir esta desventaja, la universidad podría implementar la apertura de servicios de guardería para bebés y niños, por ejemplo.

La identificación de las variables socioeconómicas asociadas de manera negativa con el RA resulta de suma utilidad para reconocer cuáles son los grupos o sectores en situación de mayor vulnerabilidad educativa (alumnas madres, alumnos que se desplazan desde localidades del interior, alumnos que trabajan a jornada completa, etc.). Y el análisis permite avanzar aún más: la estimación de la intensidad de los efectos negativos de las diversas variables permite reconocer la gravedad de la desventaja en términos educativos, es decir, ordenar las desventajas en función de su gravedad.

Contando con estas investigaciones, la universidad o facultad que se proponga atenuar las desigualdades preexistentes que resulten más nocivas para sus alumnos desaventajados podría implementar las medidas paliativas de mayor impacto, de acuerdo a sus particulares circunstancias. Sería esta una forma de optimizar la utilización de los recursos presupuestarios propendiendo a la mejora en la eficacia escolar y la igualdad de oportunidades educativas. 
Tabla 7. Comparación de Resultados UNNE con otras Universidades Nacionales

\begin{tabular}{ll}
\hline \multicolumn{1}{c}{\begin{tabular}{c}
\multicolumn{1}{c}{ RESULTADOS DE OTRAS INVESTIGACIONES } \\
NACIONALES
\end{tabular}} & \multicolumn{1}{c}{$\begin{array}{c}\text { RESULTADOS DEL PRESENTE } \\
\text { TRABAJO }\end{array}$} \\
\hline Tanto en los estudios realizados por Porto y Di & $\begin{array}{l}\text { Los alumnos varones presentan un } \\
\text { menor RA que las mujeres. }\end{array}$ \\
$\begin{array}{l}\text { Gresia (2001), y Di Gresia (2002), los varones tienen } \\
\text { menor RA que las mujeres. }\end{array}$ & \\
\hline $\begin{array}{l}\text { En el trabajo de Porto y Di Gresia (2001), el estado } \\
\text { civil soltero es un factor con influencias ambiguas en } \\
\text { virtud de cómo se construya la variable explicada y, } \\
\text { en la mayoría de los casos, con baja significación }\end{array}$ & $\begin{array}{l}\text { Los alumnos casados tienen mayor } \\
\text { RA. Se verifica para los dos géneros } \\
\text { pero con mayor intensidad para las } \\
\text { Entica. }\end{array}$ \\
$\begin{array}{l}\text { En Di Gresia (2002), el estado civil casado } \\
\text { particularmente aparece con influencia positiva en el }\end{array}$ & \\
RA, y como un factor estadísticamente significativo. & \\
\hline
\end{tabular}

Tanto en los resultados hallados en Porto y Di

Tanto en los resultados hallados en Porto y D

Gresia (2001), como en Di Gresia (2002), la escuela

secundaria es un factor explicativo estadísticamente

significativo. Estos estudios verifican una correlación

Se carece de datos.

negativa entre la formación en escuelas secundarias

públicas y RA

El cambio de residencia del alumno (mudanza) tiene

un efecto positivo sobre el rendimiento. El impacto de tener que viajar entre distintas jurisdicciones (municipalidades) para asistir a la Facultad es negativo. -Porto y Di Gresia (2001), y Di Gresia (2002)-

En contraste, para Said Rücker (2009), el cambio de residencia tiene un impacto negativo.

Los años de permanencia en la Facultad tienen impacto negativo. (Di Gresia 2002)

Porto y Di Gresia (2001) exponen que el RA disminuye con el número de horas trabajadas. Di Gresia (2002) muestra que aquellos estudiantes que trabajan tienen mayor RA. Asimismo, los estudiantes que dedican más horas al estudio, incluyendo clases, tienen mayor RA.

En Said Rucker (2009) la situación laboral ocupado tiene un fuerte impacto negativo.

Porto y Di Gresia (2001), y Di Gresia (2002), encuentran que mayor es el RA cuanto mayor es la educación del padre y de la madre.

Di Gresia (2002) sostiene que el origen del financiamiento es una variable estadísticamente significativa: el mayor rendimiento corresponde a los estudiantes que se financian con beca y aporte familiar, luego los que tienen sólo beca, luego trabajo personal y aporte familiar y finalmente aporte familiar. En todos los casos, el resultado surge de la comparación con el financiamiento originado en el trabajo personal.

En el estudio de Fazio (2004), se pone de manifiesto que la ocupación laboral tiene efectos positivos cuando se presenta en estrecha vinculación al área de estudio del alumno y en carga horaria moderada. En los demás casos, la incidencia es negativa.
Los datos sobre lugar de procedencia son incompletos para la muestra. Se carece de datos sobre viajes interjurisdiccionales.

Se carece de datos.

Se carece de los datos

correspondientes a horas de estudio de los alumnos. Utilizando como proxy la variable "situación laboral ocupado" (que implicaría menor cantidad de horas disponibles para el estudio), se observa que quienes trabajan tienen un menor RA que los alumnos inactivos. Además, mientras mayor es la carga horaria del trabajo menor es el RA.

Cuanto mayor es la educación de los padres, mayor es el RA de los alumnos.

El origen del financiamiento es una variable estadísticamente significativa: el mayor rendimiento corresponde a quienes se financian con becas, luego quienes se financian con aportes familiares, y luego quienes se financian con su propio trabajo. El financiamiento por planes sociales se asocia a rendimientos marcadamente inferiores. Las variables se toman como no excluyentes.

Se verifican los hallazgos de Fazio (2004). 


\section{Agradecimientos}

El equipo de investigación agradece a las autoridades de Rectorado y de las Facultades de Odontología y Derecho de la Universidad Nacional del Nordeste por su colaboración en la provisión de las estadísticas indispensables para los estudios realizados.

\section{Referencias}

Blanco Bosco, E. (2009). La desigualdad de resultados educativos. Aportes a la teoría desde la investigación sobre eficacia escolar. Revista Mexicana de Investigaciones Educativas, 14(43), 1019-1049.

Cascón, I. V. (2000). Predictores del rendimiento académico en alumnos de primero y segundo de BUP. Recuperado de: https://campus.usal.es/ inico/investigacion/jornadas

Cervini, R. (2002a). Desigualdades en el logro académico y reproducción cultural en la educación primaria de Argentina. Un modelo de tres niveles. Revista Mexicana de Investigación Educativa, 7(16), 445-500.

Cervini, R. (2002b). Desigualdades socioculturales en el aprendizaje de matemática y lengua de la educación secundaria en Argentina: un modelo de tres niveles. Revista Electrónica de Investigación y Evaluación Educativa, 8(2), 135-158. doi: 10.7203/relieve.8.2.4365

Cervini, R. (2003a). Diferencias de resultados cognitivos y no-cognitivos entre estudiantes de escuelas públicas y privadas en la educación secundaria de Argentina: un análisis multinivel. Education Policy Analysis Archives, 1 1(6), 234-360

Cervini, R. (2003b). Relaciones entre composición estudiantil, proceso escolar y el logro en matemáticas en la educación secundaria en Argentina. Revista Electrónica de Investigación Educativa, 5(1), 73-98.

Cervini, R. (2004). Influencia de los factores institucionales sobre el logro en matemática de los estudiantes en el último año de la educación media de Argentina. Un modelo de tres niveles. REICE. Revista Iberoamericana sobre Calidad, Eficacia y Cambio en Educación, 2(1), 5-23.

Cervini, R. y Basualdo, M. (2003). La eficacia educativa del sector público. El caso de las escuelas secundarias técnicas en Argentina. Revista Latinoamericana de Estudios Educativos, 33(3), 5392.

Cervini, R., Dari, N., Quiroz, S. y Atorresi, A. (2014). Maestro, aula y aprendizaje en América Latina. Los datos del SERCE. REICE. Revista Iberoamericana sobre Calidad, Eficacia y Cambio en Educación, 12(2), 105-137.

Coleman, J. (1967b). Equality of educational opportunity, reconsidered. Baltimore, MD: John Hopkins University.

Coleman, J. (1968a). Responsability of schools in the provision of equal educational opportunity. Baltimore, MD: John Hopkins University.

Coleman, J. (1968b). The evaluation of equality of educational opportunity. Baltimore, MD: John Hopkins University.

Coleman, J., Campbell, E. Q., Hobson C. J., McPartland J., Mood, A. M., Weinfeld, F. D. y York, R. L. (1966). Equality of educational opportunity, Washington: U.S. Department of Health, Education and Welfare, Office of Education, Government Printing Office.

Di Gresia L., Porto A. y Ripani L. (2002). Rendimiento de estudiantes de las universidades públicas argentinas. Recuperado de: http://www.depeco.econo.unlp.edu.ar/doctrab/doc45.pdf 
Edel Navarro, R. (2003). El rendimiento académico: concepto, investigación y desarrollo. REICE. Revista Iberoamericana sobre Calidad, Eficacia y Cambio en Educación, 1(2), 97-126.

Fazio, M. (2004). Incidencia de las horas trabajadas en el rendimiento académico de estudiantes universitarios argentinos. Recuperado de: http://www.depeco.econo.unlp.edu.ar

Fernández Aguerre, T. (2004). Distribución del conocimiento escolar: clases sociales, escuelas y sistema educativo en Latinoamérica. Tesis Doctoral. Centro de Estudios Sociológicos (CES). Facultad de Ciencias Sociales. Uruguay.

Fraser, B., Walberg, H., Welch, W. y Hattie, J. (1987). Syntheses of educational productivity research. International Journal of Educational Research, 11(2), 147-252. doi: 10.1016/08830355(87)90035-8

Fuller, B. (1987). School effects in the third world. Review of Educational Research, 57(3), 255-292.

Garbanzo Vargas, G. (2013). Factores asociados al rendimiento académico en estudiantes universitarios desde el nivel socioeconómico: un estudio en la Universidad de Costa Rica. Revista Electrónica Educare, 17(3), 57-87. doi: 10.15517/revedu.v31i1.1252

Hanushek, E., Kain, J. F., Markman, J. B. y Rivkin S. G. (2003). Does peer ability affect student achievement? Journal of Applied Econometrics, Empirical Analysis of Social Interactions, 18(5), 527-544. doi: 10.3386/w8502

Heyneman, S. (1976). Influences on academic achievement: A comparison of results from Uganda and more industrialized societies. Sociology of Education, 49(3), 200-211. doi: $10.2307 / 2112231$

Heyneman, S. y Loxley W. (1982). Influence on academic achievement across high and low income countries: a reanalysis of IEA data. Sociology of Education, 55(1), 13-21. doi: $10.2307 / 2112607$

Jencks, C., Smith M., Acland, H., Bane M. J., Gintis, H., Heyns B. y Michelson S. (1972). Inequality: a reassessment of the effects of family and schooling in America. Nueva York: Basic.

Llach, J. J. (2006). El desafio de la equidad educativa. Buenos Aires: Granica.

Llach, J. J. y Schumacher, F. J. (2003). Escuelas ricas para los pobres. La discriminación social en la educación primaria argentina, sus efectos en los aprendizajes y propuestas para superarla. Buenos Aires: IAE.

Murillo, F. J. (2003). Una panorámica de la investigación Iberoamericana sobre eficacia escolar. REICE. Revista Iberoamericana sobre Calidad, Eficacia y Cambio en Educación, 1(1), 1-14.

Porto, A. y Di Gresia, L. (2001). Rendimiento de estudiantes universitarios y sus determinantes. Buenos Aires: Departamento de Economía de la Universidad Nacional de La Plata.

Said Rücker, P., Chiapello, J. A. y Espíndola de Markowsky, M. E. (2009). Condicionantes socioeconómicos para la aprobación de la primera asignatura de la carrera de Medicina. Educación Médica Superior, 23(4),185-193.

Sorensen, A. B., Dumais S. A. y Stephen L. M. (1999). Schools, learning and educational opportunity: a replication of the main conclusions of the equality of educational opportunity report. Boston, MA: Harvard University Press.

Wooldridge, J. M. (2002). Introductory econometrics: a modern approach. Cincinnati, OH: Southwestern College Publishing. 
Anexo 1. Descripción de variables utilizadas

\begin{tabular}{|c|c|c|c|}
\hline GRUPO & VARIABLE & DESCRIPCIÓN & VALORES \\
\hline \multirow{4}{*}{$\begin{array}{l}\text { Perfil } \\
\text { General }\end{array}$} & Unidad académica & Unidad académica a la que pertenece el alumno & Nombre de la Unidad Académica \\
\hline & Sexo & Sexo del alumno & O: Masculino; 1: Femenino \\
\hline & Edad_est & Edad estimada del alumno & Numérico (en años) \\
\hline & Traslado & $\begin{array}{l}\text { Relación entre residencia de origen y residencia durante } \\
\text { período lectivo del alumno }\end{array}$ & $\begin{array}{l}\text { 1: Ciudad de origen distinta a la residencia durante ciclo } \\
\text { lectivo }\end{array}$ \\
\hline \multirow{3}{*}{$\begin{array}{l}\text { Familia } \\
\text { Núcleo }\end{array}$} & Estado Civil & Estado civil del alumno & 1: Soltero, 2: Casado, 3: Otros \\
\hline & Hijos & Cantidad de hijos del alumno & Numérico \\
\hline & Familiares a cargo & Cantidad de familiares a cargo del alumno & Numérico \\
\hline \multirow{5}{*}{$\begin{array}{l}\text { Perfil } \\
\text { Laboral }\end{array}$} & Situación laboral & Situacion laboral del alumno & 1: Ocupado; 2: Desocupado; 3: Inactivo \\
\hline & Tipo de ocupación & Tipo de ocupación de los alumnos ocupados & 1: Patrón; 2: Cuenta propia; 3: Empleado; 4:Pasante \\
\hline & Descuento jubilatorio & Realización de aportes jubilatorios de los alumnos ocupados & 1: Realiza aportes jubilatorios \\
\hline & Horas de trabajo & Horas semanales trabajadas por los alumnos ocupados & $\begin{array}{l}\text { 1: Hasta } 10 \text { horas; } 2 \text { : Más de } 10 \text { y hasta } 20 \text {; } 3 \text { : Más de } 20 \text { y } \\
\text { hasta } 35 \text {; } 4 \text { : Más de } 35 .\end{array}$ \\
\hline & Rel. trabajo-carrera & Relación entre la ocupación y los estudios en los alumnos & 1: Sin relación; 2: Relac. Parcial; 3: Relac. Total \\
\hline \multirow{4}{*}{$\begin{array}{l}\text { Nivel } \\
\text { Socio- } \\
\text { económico }\end{array}$} & Cobertura médica & Tipo de cobertura médica del alumno & $\begin{array}{l}\text { O: Carece de cobertura; 1: Obra social por ser familiar a cargo; } \\
\text { 2: O.S. por su propio trabajo; 3: O.S. o prepaga por afiliación } \\
\text { voluntaria; 4: Cobertura otorgada por la Univ. }\end{array}$ \\
\hline & Costeo de estudios & $\begin{array}{l}\text { Forma en que se costea los estudios el alumno (opciones no } \\
\text { excluyentes) }\end{array}$ & $\begin{array}{l}\text { 1: Aportes familiares; 2: Trabajo propio; 3: Becas; 4: Planes } \\
\text { sociales; 5: Otras }\end{array}$ \\
\hline & Beneficiario de becas & Condición de beneficiario de becas & 1: Es beneficiario de becas \\
\hline & $\begin{array}{l}\text { Cantidad de Becas } \\
\text { Universitarias }\end{array}$ & Cantidad de becas universitarias de las que es beneficiario & Numérico \\
\hline \multirow{3}{*}{$\begin{array}{l}\text { Familia de } \\
\text { Origen }\end{array}$} & $\begin{array}{l}\text { Nivel de instrucción de } \\
\text { padre/madre }\end{array}$ & Máximo nivel de instrucción alcanzado por el padre/madre & $\begin{array}{l}\text { O: Sin instrucción o con Primario Incompleto; 1: Primario } \\
\text { Completo o Secundario Incompleto; 2: Secundario Incompleto } \\
\text { o Terciario/Universitario Incompleto; 3: } \\
\text { Terciario/Universitario Completo. }\end{array}$ \\
\hline & $\begin{array}{l}\text { Situación laboral de } \\
\text { padre/madre }\end{array}$ & Situación laboral de padre/madre & 1: Ocupado; 2: Desocupado; 3: Inactivo \\
\hline & $\begin{array}{l}\text { Tipo de ocupación de } \\
\text { padre/madre }\end{array}$ & Tipo de ocupación de los padres ocupados & 1: Patrón; 2: Cuenta propia; 3: Empleado; 4:Pasante \\
\hline
\end{tabular}




\section{Anexo 2. Observaciones perdidas por variable}

\begin{tabular}{|c|c|c|c|}
\hline VARIABLE & $\mathbf{N}$ & PERDIDOS & \%* \\
\hline Promedio & 9.694 & 662 & 6,4 \\
\hline Edad_est & 10.339 & 17 & 0,2 \\
\hline sexo & 10.356 & $\mathrm{O}$ & 0,0 \\
\hline estado_civil & 9.877 & 479 & 4,6 \\
\hline cant_hijos_alum & 10.356 & $\mathrm{O}$ & 0,0 \\
\hline cant_fam_cargo & 7.451 & 2.905 & 28,1 \\
\hline tiene_fliaohijo & 7.451 & 2.905 & 28,1 \\
\hline alu_obra_social & 8.224 & 2.132 & 20,6 \\
\hline traslado & 5.962 & 4.394 & 42,4 \\
\hline alu_cos_est_ap_fam & 10.300 & 56 & 0,5 \\
\hline alu_cos_est_trab & 10.300 & 56 & 0,5 \\
\hline alu_cos_est_beca & 10.300 & 56 & 0,5 \\
\hline alu_cos_est_plsoc & 10.300 & 56 & 0,5 \\
\hline alu_cos_est_otra & 10.300 & 56 & 0,5 \\
\hline tiene_beca & 10.087 & 269 & 2,6 \\
\hline cant_becas_uni & 10.276 & 80 & 0,8 \\
\hline existe_trab_alum & 10.333 & 23 & 0,2 \\
\hline alu_trab_hace & 4.153 & 6.203 & 59,9 \\
\hline alu_trab_desjub & 10.069 & 287 & 2,8 \\
\hline est_padre & 1.269 & 9.087 & 87,7 \\
\hline sit_laboral_padre & 7.208 & 3.148 & 30,4 \\
\hline padre_trab_hace & 6.090 & 4.266 & 41,2 \\
\hline est_madre & 1.285 & 9.071 & 87,6 \\
\hline sit_laboral_madre & 8.365 & 1.991 & 19,2 \\
\hline madre_trab_hace & 5.137 & 5.219 & 50,4 \\
\hline
\end{tabular}

Nota: * Los porcentajes se calcularon sobre el total de observaciones de cada variable. Fuente: Elaboración propia. 


\section{Anexo 3. Análisis por unidades académicas. UNNE}

\begin{tabular}{|c|c|c|c|c|}
\hline & ODO & GÍA & & \\
\hline & B & Sig. & B & Sig. \\
\hline (Constante) & 10,15 & ****** & 5,13 & 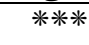 \\
\hline sexo & $-0,02$ & & 0,19 & ***** \\
\hline Edad_est & $-0,17$ & ****** & 0,06 & **** \\
\hline Edad_est_2 & 0,00 & ****** & 0,00 & ***** \\
\hline EC_casado & 0,15 & & 0,13 & ***** \\
\hline EC_otros & 0,56 & * & 0,01 & \\
\hline cant_hijos_alum & 0,01 & & $-0,08$ & ***** \\
\hline sit_lab_ocup & $-0,07$ & & $-0,15$ & ***** \\
\hline sit_lab_desocup & 0,02 & & $-0,17$ & ***** \\
\hline OS_fam & 0,08 & & 0,11 & ***** \\
\hline OS_trab & 0,27 & & 0,14 & ***** \\
\hline OS_afil & 0,23 & * & 0,22 & **** \\
\hline OS_uni & $-0,03$ & & 0,25 & ***** \\
\hline alu_cos_est_ap_fam & 0,05 & & 0,14 & ***** \\
\hline alu_cos_est_trab & $-0,02$ & & 0,04 & \\
\hline alu_cos_est_beca & 0,06 & & 0,11 & \\
\hline alu_cos_est_plsoc & $-1,85$ & *** & $-0,25$ & *** \\
\hline alu_cos_est_otra & $-0,03$ & & $-0,03$ & \\
\hline cant_becas_uni & 0,07 & & 0,02 & \\
\hline $\mathrm{N}$ & & & & \\
\hline $\mathrm{R}^{2}$ corregido & & & & \\
\hline
\end{tabular}

\section{Breve CV de los autores}

\section{Carlos Cristóbal Coschiza}

Licenciado en Economía egresado de la Facultad de Ciencias Económicas de la Universidad Nacional del Nordeste (Argentina). Especialista en Preparación y Evaluación de Proyectos egresado de la Universidad Tecnológica Nacional (Argentina). Es docente en la Facultad de Ciencias Económicas de la Universidad Nacional del Nordeste en las cátedras de Microeconomía I y Macroeconomía I. Consultor empresarial en el ámbito privado, trabajó en el Área de Vinculación y Transferencia Tecnológica de la Universidad Nacional del Nordeste teniendo a su cargo el área de proyectos de innovación productiva, fue también responsable del departamento de proyectos de la empresa Fiduciaria del Norte SA. En la actualidad es Subsecretario de Información y Estadística del Municipio de la Ciudad de Resistencia (Provincia del Chaco, Argentina). Email: carloscoschiza@gmail.com

\section{Juan Martín Fernández}

Licenciado en Economía por la Universidad Nacional del Nordeste (UNNE, Argentina). Máster en Desarrollo Económico e Innovación por la Universidad de Santiago de Compostela (España), a través de la beca Erasmus Mundus. Maestrando del Máster en Gobierno y Economía Política de la Escuela de Gobierno de la Provincia del Chaco 
(Argentina). Se desempeña como profesor de Política Económica y Microeconomía II en la Facultad de Ciencias Económicas de la UNNE y como Subsecretario de Desarrollo Económico de la Municipalidad de la Ciudad de Corrientes (Argentina). Fue investigador del Consejo Económico y Social de la Provincia del Chaco. Ha investigado sobre los determinantes familiares y escolares de los resultados educativos en distintos niveles educativos. También ha investigado sobre mercado laboral, empleo industrial, innovación, negociaciones salariales y análisis de políticas públicas. Email: jmfernandez0oo@gmail.com

\section{Guillermo Gapel Redcozub}

Es Licenciado en Economía (2004) y Abogado (2007) por la Universidad Nacional del Nordeste (UNNE, Argentina). Especialista en Docencia Universitaria por la Facultad de Humanidades de la UNNE y Master of Laws (LLM) por la Escuela de Leyes de la Universidad de Harvard (2012). Es Profesor Titular Regular de la Asignatura Derecho Civil IV (Derechos Reales) en la Facultad de Derecho de la UNNE. Ex becario Fulbright (2011/2). Fue investigador del Consejo Económico y Social de la Provincia del Chaco (Argentina). En distintos ámbitos, ha investigado sobre la igualdad de oportunidades educativas, los factores determinantes del rendimiento académico y la enseñanza del derecho en contextos de matrícula numerosa. Es autor y evaluador de artículos en diversas publicaciones internacionales. Email: guillermogapel@gmail.com

\section{Marcelo E. Nievas}

Licenciado en Economía de la Universidad Nacional del Nordeste (UNNE, Argentina) en el año 2004. Magister en Desarrollo Local de la Universidad Nacional San Martín (Argentina) y la Universidad Autónoma de Madrid (España) en el año 2014; realizó el Programa de Desarrollo Empresarial del IAE Business School (Argentina) en el 2015. Es docente universitario desde 2004, actualmente Profesor Adjunto de Macroeconomía I de la Facultad de Ciencias Económicas de la UNNE. Fue Docente de Macroeconomía la Universidad Nacional del Chaco Austral (Argentina) y Universidad de la Cuenca del Plata (Argentina).

En su trayectoria profesional se destacan labores en el sector público (Comisión Nacional de Valores de Argentina), privado (Bolsa de Comercio de la Provincia del Chaco) y organizaciones no gubernamentales (Consejo Económico y Social de la Provincia del Chaco, Sistema Previsional y Social para Profesionales de Ciencias Económicas). Participa de grupos de investigación y docencia en temas vinculados a finanzas y desarrollo económico. Email: menievas@gmail.com

\section{Haraví E. Ruiz}

Abogada por la Universidad Nacional del Nordeste (UNNE, Argentina) en 2007. Especialista en Docencia Universitaria por la Facultad de Humanidades de la UNNE y en Teoría y Técnica del Proceso Judicial por la Facultad de Derecho de la UNNE. Miembro por concurso de la Cátedra de Instituciones de Derecho Privado II de la Facultad de Ciencias Económicas y Miembro Ad-Honorem de la Cátedra de Derecho Agrario, Ambiental, de la Minería y Energía de la Facultad de Derecho, Ciencias Sociales y 
Políticas, ambas de la Universidad Nacional del Nordeste (UNNE). Ex-Becaria de Investigación de Pre-grado y Posgrado de la Universidad Nacional del Nordeste, Ex Becaria PHD Erasmus Mundus. Actualmente se desempeña como Becaria Exclusiva de Doctorado del Consejo Nacional de Investigaciones Científicas y Técnicas (CONICET) y es doctoranda del Doctorado en Ciencias Jurídicas de la Facultad de Derecho de la UNNE. Fue y es miembro de diversos programas de Extensión Universitaria en el área de Desarrollo y Asociatividad Rural, y de Proyectos de Investigación Multidisciplinarios en el ámbito del Derecho Agrario y Ambiental y el Desarrollo Rural. Focaliza sus investigaciones en las áreas de Enseñanza del Derecho, Derecho Agrario y Ambiental. Email: haraviruiz@gmail.com 